\section{Geography lesson in dry AMD}

\section{By Tim Fulmer, Senior Writer}

American researchers have identified an RNA-based mechanism in the retina that could be responsible for triggering the blindness associated with advanced dry age-related macular degeneration. ${ }^{1}$ The team is developing inhibitors and ultimately hopes to submit an IND.

Unlike wet AMD, which results from scarring caused by leaky blood vessels and thus can be treated with a variety of angiogenesis inhibitors, dry AMD is initially characterized by the buildup of extracellular debris beneath the retina. Over time, those deposits cause significant atrophy of the retinal pigment epithelial (RPE) layer-a condition known as geographic atrophy - and can ultimately advance to permanent blindness.

Although difficult to predict, dry AMD can turn into wet $\mathrm{AMD}$ at any time during disease progression. There are no medical or surgical treatments for dry AMD.

To find potential targets for geographic atrophy, Jayakrishna Ambati and colleagues

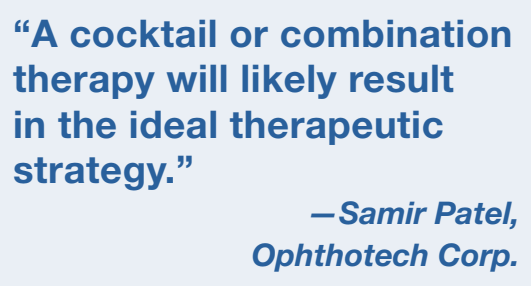

\section{Targeting Alu}

In cultured human RPE cells with low DICER1 levels, antisense oligonucleotides targeting Alu RNA significantly blocked RPE cellular degeneration compared with control oligonucleotides $(p<0.05)$.

The findings were published in Nature.

Ambati told SciBX that he now plans to explore "molecular platforms for Alu inhibition, including the Alu antisense therapy described in the paper. Once we settle on a lead candidate, we plan to proceed to GMP manufacture and FDA IND filing."

Although the University of Kentucky researchers initially plan to test Alu inhibitors as monotherapy for dry AMD, the heterogeneous nature of the disease makes it unlikely that targeting only Alu RNA would be sufficient to stave off blindness.

"Chronic diseases such as dry AMD are very heterogeneous and progress through a series of early and late stages. The advanced stage of dry AMD is characterized as either geographic atrophy or conversion to neovascular disease," said Samir Patel, cofounder, president and CEO of Ophthotech Corp. "As a result, different molecular mechanisms may predominate at different times, and a single therapeutic target may not be useful for all stages. A cocktail or combination therapy will likely result in the ideal therapeutic strategy."

He added: "It is important to use preclinical animal models, together with human histopathology and genetic studies, if possible, to better understand the role of Alu RNA in the overall pathogenesis and progression of dry AMD and geographic atrophy," he continued. "This information should give us a better at the University of Kentucky College of Medicine initially looked at levels of proteins and nucleic acids in eye samples from dry AMD patients. Ambati is vice chair of ophthalmology and visual sciences at the college.

The researchers found that levels of the microRNA-processing enzyme dicer 1 ribonuclease type III (DICER1) were significantly lower in patient eyes than in non-AMD control eyes $(p=0.0036)$. DICER1 levels were unchanged in the RPE layer of human eyes with other retinal diseases, suggesting that low DICER1 could be a specific marker of geographic atrophy.

The team next generated Dicer 1 knockout mice to recapitulate the geographic atrophy phenotype in animals. All the Dicer 1 knockouts showed degeneration of the RPE layer compared with wild-type littermate controls.

Subsequent studies in human RPE cells and mice revealed the specific mechanism by which low DICER1 levels led to degeneration of the RPE cells or layer, respectively. The decrease in DICER1 caused the accumulation of cytotoxic Alu RNA molecules in the RPE layer, where they caused degeneration of tissues making up the retina and macula. Alu RNAs are retrotransposon sequences that exist throughout the human genome but do not code for proteins. idea of how best to pair an Alu RNA-targeting strategy with other complementary mechanisms in dry AMD."

Ophthotech's ARC1905, a selective inhibitor of complement component 5 , is in Phase IIa testing to treat advanced dry AMD with geographic atrophy. The company is targeting the complement system "because there is strong evidence that excessive inflammation is important in AMD and that local inflammation and activation of the complement cascade drives formation of crystalline deposits beneath the retina that are the hallmark of dry AMD," said Patel.

Ryo Kubota, president and CEO of Acucela Inc., noted that it's still unclear what causes the initial decreases of DICER1 levels that lead to high amounts of Alu RNA. "Our research suggests that the underlying pathology of AMD can be traced to oxidative stress caused by toxic byproducts of the visual cycle, which negatively impact human RPE cells," said Kubota.

Acucela's ACU-4429, a small molecule that targets the retinal pigment epithelium-specific protein $65 \mathrm{kDa}$ (RPE65) to modulate the visual cycle, is in Phase II testing to treat dry AMD.

Ambati thinks targeting Alu RNA might have advantages over the other strategies. "Compared with complement inhibitors, which modulate the immune system, targeting Alu RNA could have less 


\section{ANALYSIS}

potential to increase the patient's risk of infection. And compared with visual cycle inhibitors, an anti-Alu RNA approach might have lower rates of nontolerance by avoiding the negative effects visual cycle inhibitors can have on night vision," he said.

Ambati said the University of Kentucky has filed multiple patent applications covering the paper's findings and the IP is available for licensing.

Fulmer, T. SciBX 4(8); doi:10.1038/scibx.2011.211

Published online Feb. 24, 2011
REFERENCES

1. Kaneko, H. et al. Nature; published online Feb. 6, 2011; doi:10.1038/nature09830

Contact: Jayakrishna Ambati, University of Kentucky College of Medicine, Lexington, Ky.

e-mail: jamba2@email.uky.edu

\section{COMPANIES AND INSTITUTIONS MENTIONED}

Acucela Inc., Seattle, Wash.

Ophthotech Corp., Princeton, N.J.

University of Kentucky College of Medicine, Lexington, Ky. 